\title{
Héctor Rubinstein and the start of Swedish Neutrino Astronomy
}

\author{
Per Olof Hulth* \\ Oskar Klein Center, Department of Physics, Stockholm University \\ Address, Roslagsbacken 21, Stockholm, Sweden, SE-10691 Stockholm Sweden \\ E-mail: hulthefysik.su.se
}

In the beginning of 1990, Swedish research groups started to get interested in the search for neutrinos from the Cosmos. These neutrinos could be the key to answer so far unsolved questions such as where the high energy cosmic rays are coming from and what the dark matter in the universe consists of. Héctor Rubinstein pushed this idea and he managed to get several Swedish scientists interested. Héctor played a very important role in the development from the original idea to today's strong Swedish involvement in the huge IceCube neutrino telescope at the South Pole. This paper describes the development in Sweden of this new field of Science.

Quarks, Strings and the Cosmos - Héctor Rubinstein Memorial Symposium August 09-11, 2010

AlbaNova Stockholm Sweden

\footnotetext{
* Speaker.
} 


\section{Introduction}

Héctor Rubinstein played an important role in the discussions about modernising Swedish science. He had strong opinions about high quality science. In the beginning of the 1990-ties we started to discuss the fast developments in the field of neutrino astronomy. The possibility to search for the sources of the highest energy cosmic rays and the search for dark matter annihilation in the centre of the Earth and the Sun by using neutrinos was intriguing. The possibility to build a large neutrino detector in Sweden came up and it was the start for an intense activity in the field which today is reflected in Sweden's large participation in the huge IceCube neutrino telescope at the South Pole, Antarctica. The development was not always moving forward. In this paper we give a brief description about the history leading to IceCube.

\section{Why detect neutrinos from Space?}

Cosmic rays (CR) were discovered almost 100 years ago but we have not yet been able to identify any source by direct observation. The flux of cosmic rays has been measured up to an incredible energy of $10^{20} \mathrm{eV}$. The acceleration process for these energies is not completely understood. The cosmic rays are expected to interact with matter or with the radiation field in the vicinity of the source creating hadrons which decay to neutrinos. The neutrinos will traverse the universe without being absorbed by matter or deflected by magnetic fields. The expected flux of cosmic neutrinos can be estimated from the observed cosmic ray flux. The neutrino flux is predicted to be low which demands a very large detector mass in order to get enough interacting neutrinos. To get enough mass one has to use cheap natural detector materials like e.g. water or ice. The interacting neutrinos produce charged secondary particles which generate Cherenkov light when traversing the detector medium. The muons produced by muon neutrinos will for high energy keep the direction of the incoming neutrinos within a degree. By equipping the medium with light detectors the arrival time and intensity of the Cherenkov light will give the necessary information to calculate the direction of the incoming neutrino.

Neutrino telescopes are exposed to an intense flux of atmospheric muons (depending on the depth of the telescope) produced by the CR interactions in the atmosphere, and the atmospheric neutrinos produced in the same interactions will be a background for extraterrestrial neutrinos. The ratio between the number of down-going atmospheric muons to the number of registered atmospheric neutrinos is about $10^{6}$.

The Earth is opaque for $v_{\mu}$ and $v_{e}$ neutrinos with energies above $10^{14} \mathrm{eV}$, giving the acceptance for $\mathrm{PeV}$ neutrinos around the direction of the horizon. For EeV neutrinos the acceptance is even above the horizon. The $\tau$ neutrinos will also interact in the Earth but the produced taus will decay before interacting, allowing the $v_{\tau}$ to continue through the Earth but at a lower energy.

\section{The PAN consortium}

A consortium of Swedish scientists was created in 1990, called Particle Astrophysics in Norrland (PAN), in order to investigate the possibility to build a neutrino telescope in Sweden. Norrland is the northern part of Sweden which was for several reasons considered a possible location for a 
telescope. The Swedish Research Council approved money for a two-year study. The consortium consisted of both experimental and theoretical groups given in table 1 .

\begin{tabular}{|c|c|c|}
\hline \multicolumn{3}{|c|}{ Particle Astrophysics in Norrland 1991-1993 } \\
\hline Experimental Physics & Theoretical Physics & Astronomy \\
\hline Manne Siegbahn institute & Stockholm University & Stockholm University \\
\hline - Prof. P. Carlson & - Doc. L. Bergström & - Prof C. Fransson \\
\hline - Dr. S. Johansson & Uppsala University & Uppsala University \\
\hline Stockholm University & - Prof. H. Rubinstein & - Prof. B Gustafsson \\
\hline - Dr. B. Erlandsson & - Dr. P. Gondolo & \\
\hline - Doc. P. O. Hulth & Royal Institute of Technology & \\
\hline - Doc. C. Walck & - Doc. H. Snellman & \\
\hline Uppsala University & Manne Siegbahn institute & \\
\hline - Doc. O. Botner & - Doc. R. Liotta & \\
\hline - Doc. S. Carius & Luleå University & \\
\hline - Doc. A. Hallgren & - Prof. S. Fredriksson & \\
\hline
\end{tabular}

Table 1: Members of the PAN consortium

The neutrino telescope first discussed was a surface detector similar to the proposed GRANDE telescope in a quarry in Arkansas, USA [1, 3]. The GRANDE detector is shown in Figure 1 and consists of five optically isolated chambers equipped with photo multipliers. The detector was designed to detect the Cherenkov light emitted from neutrinos interacting from below and gamma ray showers from above. The huge background of down going muons from cosmic ray interactions in the atmosphere had to be rejected to the order of one in $10^{10}$.

The most promising place to put a Swedish neutrino detector of this kind was the lake Torne Träsk west of the city of Kiruna. The lake is one of the most transparent in Sweden and has a maximum depth of $168 \mathrm{~m}$. There are good communications and infrastructure in Abisko where the Royal Swedish Academy of Sciences had a research station at the shore of the lake.

The project became publicly known thanks to a collaboration between the astronomer Bengt Gustafsson (PAN) and the Stockholm City Theatre (Stadsteatern) where a very successful play was set up named "Big Science - is it worth it?" It was about the detector, the scientific questions and how the local Sami population would react to

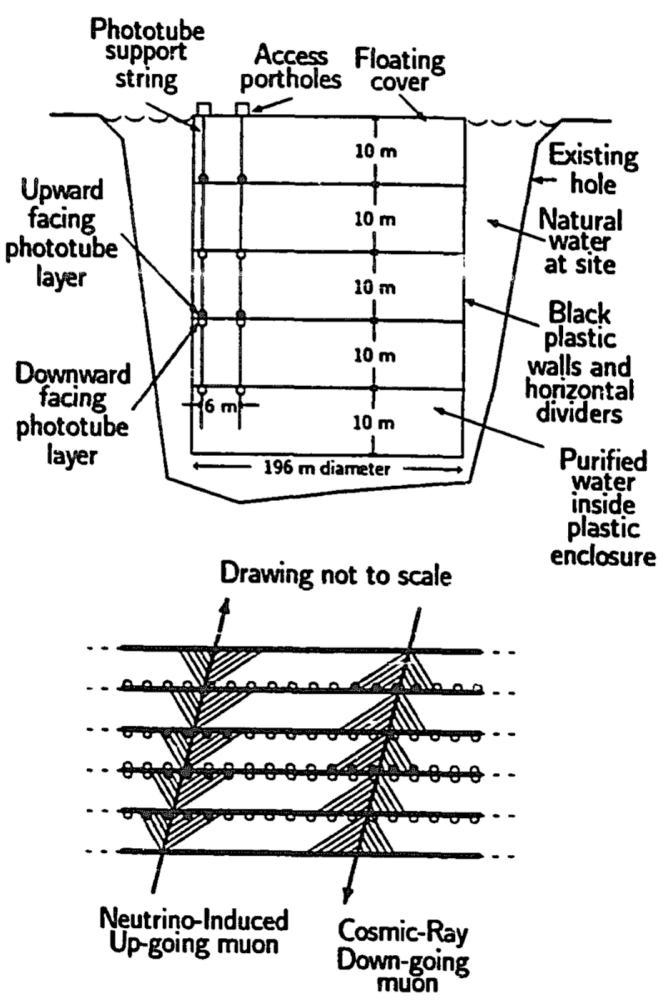

Figure 1: The proposed GRANDE detector. 
it.

We made detailed simulations of the detector geometry with different spacings of photomultipliers and different water transparencies. The astronomers in Uppsala developed a dedicated device, Saiva, for measuring the water quality in situ. However, we were not fully satisfied with its operation and rented a device from The Swedish Defence Research Agency (FOA). The attenuation length was measured in situ during several expeditions and it was found that the water quality was unfortunately (or fortunately?) not good enough which forced us to abandon Torne Träsk as a site for a Swedish neutrino telescope. Nevertheless, it is a spectacularly beautiful location.

We then started to look for interesting neutrino projects outside Sweden. Two projects were more interesting than others, DUMAND and AMANDA.

The DUMAND detector was to be deployed at $4000 \mathrm{~m}$ depth in the sea off the Big Island in Hawaii. Members of the PAN group including Héctor visited the DUMAND group in Hawaii and discussed with John Learned and others. Stockholm University contributed to the DUMAND project by providing one engineer for a few months for designing and building the optical module boards for one European DUMAND string to be deployed.

The AMANDA project was going to use the transparent ice deep under the Amundsen Scott station at the geographical South Pole in Antarctica as detector medium. In February 1991 Lars Bergström and P.O. Hulth contacted Francis Halzen at the Neutrino Telescope workshop in Venice for a first discussion. The US groups involved in the project at that time were the University of California, Berkeley, the University of California, Irvine and the University of Wisconsin, Madison. The AMANDA group had done a successful test in an existing drilled hole in Greenland in 1990 [3] by measuring the atmospheric muon flux.

After intense discussions in the PAN group in which Héctor played a significant role it was decided to join the AMANDA project. In November 1992 the Swedish Knut \& Alice Wallenberg Foundation approved an application $(1.5 \mathrm{Mkr})$ from the PAN consortium for building one detector string to be deployed in the AMANDA detector. In April 1993 an additional 2.7 Mkr were approved for more strings. The Swedish Research Council was at that time not inclined to contribute any money to the new research field in spite of the fact that they had funded the PAN studies.

\section{Glacier ice as detector medium for neutrino detectors}

The ice in a glacier is not frozen water but packed snow. When the snow accumulates over a long time and under enough pressure it transforms to ice with air bubbles. At high enough pressure and low enough temperature, a first order phase transition takes place from air-bubbles to clathratehydrates where the air molecules are trapped inside cages of hydrogen bonded water molecules. The ice clathrate is a crystalline material which is optically transparent without air bubbles. The ice sheet at the South Pole is about $2820 \mathrm{~m}$ deep and moves towards the sea with a speed of $10 \mathrm{~m}$ per year.

The ice sheet acts as a memory of atmospheric conditions over time. Dust concentrations in the atmosphere accumulate on the surface of the snow and will be embedded together with the new snow. The accumulated dust will then show up as dust layers in the ice. The ice at $2500 \mathrm{~m}$ depth at the South Pole is about 90000 years old. 
A neutrino detector in ice has to be placed deep enough so the phase transition from ice and air bubbles to the transparent clathrate hydrates has completely taken place. For the 1993 AMANDA project the required depth was thought to be $800 \mathrm{~m}$ at the South Pole location.

\section{Hot water drilling}

In order to deploy the light detectors deep in the ice one uses a drilling technique based on hot water. This was an established technique but was developed even further to fulfill the demands of making holes for a neutrino detector. The drill makes a deep hole with a diameter of about $60 \mathrm{~cm}$ with a water level about $80 \mathrm{~m}$ below the snow surface. The deployment of the detector string with the optical modules is done when the drill has been removed from the hole. The hot water drilling team for AMANDA was lead by the famous US drill leader Bruce Koci (1943-2006).

\section{The AMANDA-A detector}

The first design for AMANDA was a detector consisting of nine strings with 20 optical modules each to be deployed at $800 \mathrm{~m}-1000 \mathrm{~m}$ depth where the ice was expected to be transparent enough. The 1993 AMANDA detector is shown in Figure 2. The optical module consisted of a 10" glass sphere (Benthos) with an aluminium spacer between the half spheres. The photomultiplier was an 8" Thorn EMI and the PMT signal was read out via coaxial cables to the surface. Each optical module had a nylon diffuser sphere mounted below it connected to the surface with an optical fibre. The time calibration was done by sending a short laser pulse via the optical fibre to the module. The ice properties could be investigated by observing the emitted light from one optical module by optical modules at other strings.

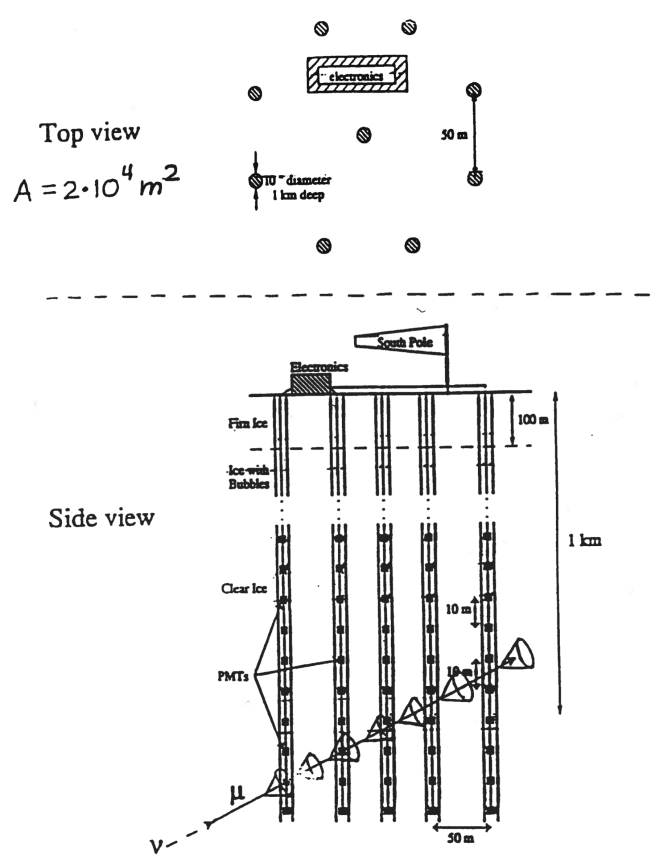

Figure 2: The AMANDA-A detector

The Swedish groups built and tested about 30 optical modules at the universities and Héctor took part in the work by closing one module himself.

The Swedish Polar Research Secretariat supported two drillers, one of them female, who became the first Swedish woman (Cecilia Malmström) at the South Pole. The drilling and deployment of the detector strings was done outdoors and the work conditions were quite rough with temperatures down to below $-40^{\circ} \mathrm{C}$. The time to drill one hole down to $1000 \mathrm{~m}$ was about $90 \mathrm{~h}$. Four strings were successfully deployed during the 1993/1994 season with a total of 80 optical modules.

When we started to investigate the ice transparency between the deployed strings by sending down laser light in the optical fibre we realised immediately that something was very, very wrong. Instead of getting a sharp time distribution corresponding to the expected travel time for photons 
going from one string to another we got a broad distribution with light pulses as late as $1500 \mathrm{~ns}$. This corresponds to light traveling $300 \mathrm{~m}$ in the ice, instead of the $20 \mathrm{~m}$ that was the distance between the strings.

The absorption length in the ice was estimated from the timing distributions to be about $60 \mathrm{~m}$ for a photon wave length of $515 \mathrm{~nm}$ which was surprisingly much longer than expected. It was more than twice the best value for laboratory ice. However, the scattering length was only a few tens of $\mathrm{cm}$ instead of the expected several tens of metres. We had a lot of air bubbles still at a depth of $1000 \mathrm{~m}$ ! The scattering length increased as a function of depth between $800 \mathrm{~m}$ and $1000 \mathrm{~m}$ indicating that the density of air bubbles decreased with depth. The observed optical properties were published in Science [4].

\section{The AMANDA-II detector}

We were in a really critical situation with a detector placed in the wrong place. There were however two very interesting observations: 1 ) the absorption length of the light was much longer than expected, 2) the density of bubbles decreased as a function of depth. During the following season (1994/1995 austral summer), the Swedish groups made new measurements of the ice properties at different wavelengths in the AMANDA-A ice. We observed strong wavelength dependence of the absorption length and measured an astonishing $225 \mathrm{~m}$ absorption length at $420 \mathrm{~nm}$. That is a factor of ten better than expected. The ice between the air bubbles had fantastic properties.

A new AMANDA detector to be situated at depths below $1500 \mathrm{~m}$ was designed and the K\&A Wallenberg foundation approved additional $2 \mathrm{Mkr}$ for continuing AMANDA. A German group from DESY Zeuthen joined 1994 the AMANDA collaboration. They had experience from the neutrino experiment in Lake Baikal.

We started to deploy the first strings of AMANDA-II in December 1995. We had made a design of the detector assuming a specific scattering length. When we had deployed three strings we realised that we had been too optimistic about the scattering length so we decided on the ice to

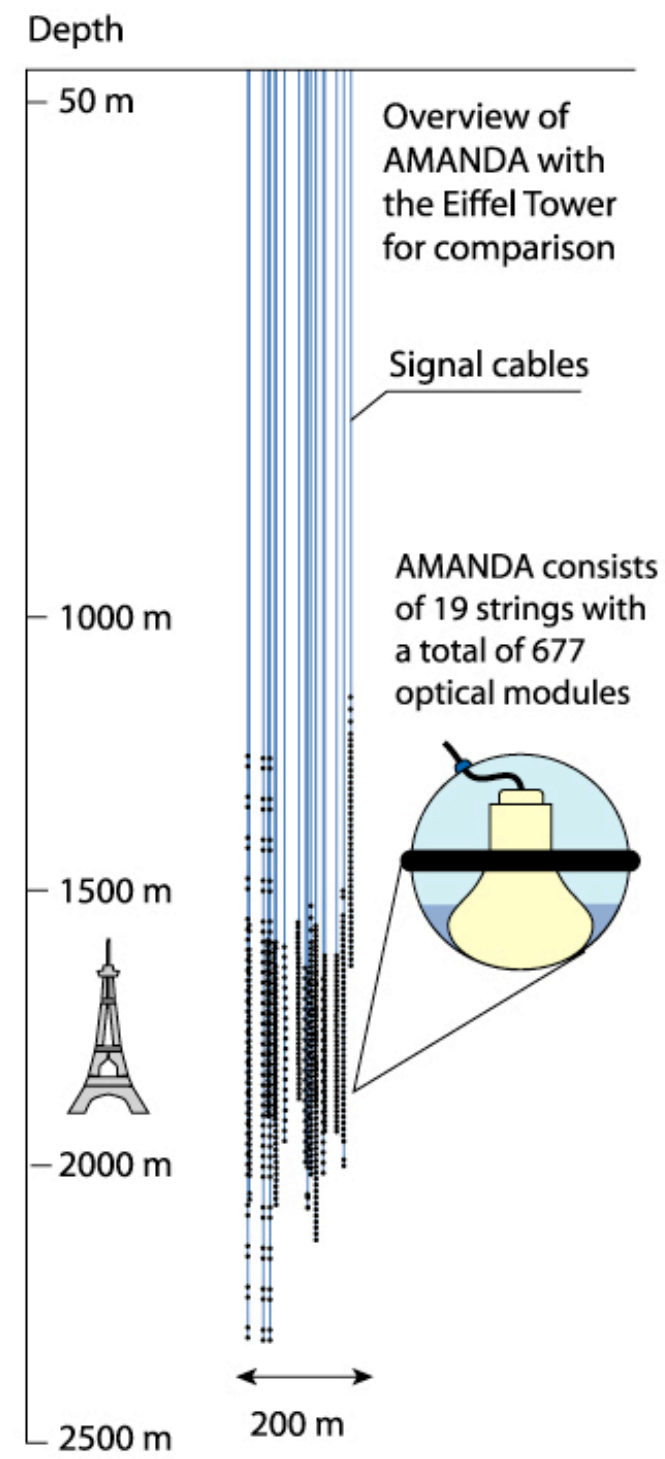

Figure 3: The AMANDA-II detector with 19 strings completed in the year 2000. 
put the fourth string in-between the first three strings (Héctor first interpreted this to be a complete disaster again, but this time Héctor was wrong!).

The four deployed strings turned out to be a fully working neutrino detector capable of rejecting down-going atmospheric muons despite a very tricky geometry (400 $\mathrm{m}$ in height and 100 $\mathrm{m}$ in diameter). We detected the first up-going neutrinos with these four strings. We applied for building a larger detector and received 7.5 Mkr from the K\&A Wallenberg foundation. Six additional strings were deployed in the 1995/1996 season giving a 10-string detector. In the next season we deployed an additional three strings and part of the optical modules were deployed above and below the main detector at depths down to $2350 \mathrm{~m}$ in order to investigate the quality of the ice. We also deployed one camera system made at Stockholm University at the bottom of string 13 which recorded the deployment and the refreezing of the hole at $2350 \mathrm{~m}$ depth. An additional six strings were deployed in 1999/2000, completing the AMANDA-II detector with in total 19 strings. One string (number 17) was stuck in the hole and did not reach the planned depth. Sweden designed and built the trigger for this detector and designed and constructed the pre-amplifiers for the PMT signals .

A paper was published in Nature [5] based on the 10 string detector showing the feasibility of using the Antarctic ice as detector medium. That was the last paper in AMANDA which Héctor signed. He then followed the development very closely but not as a member of the collaboration.

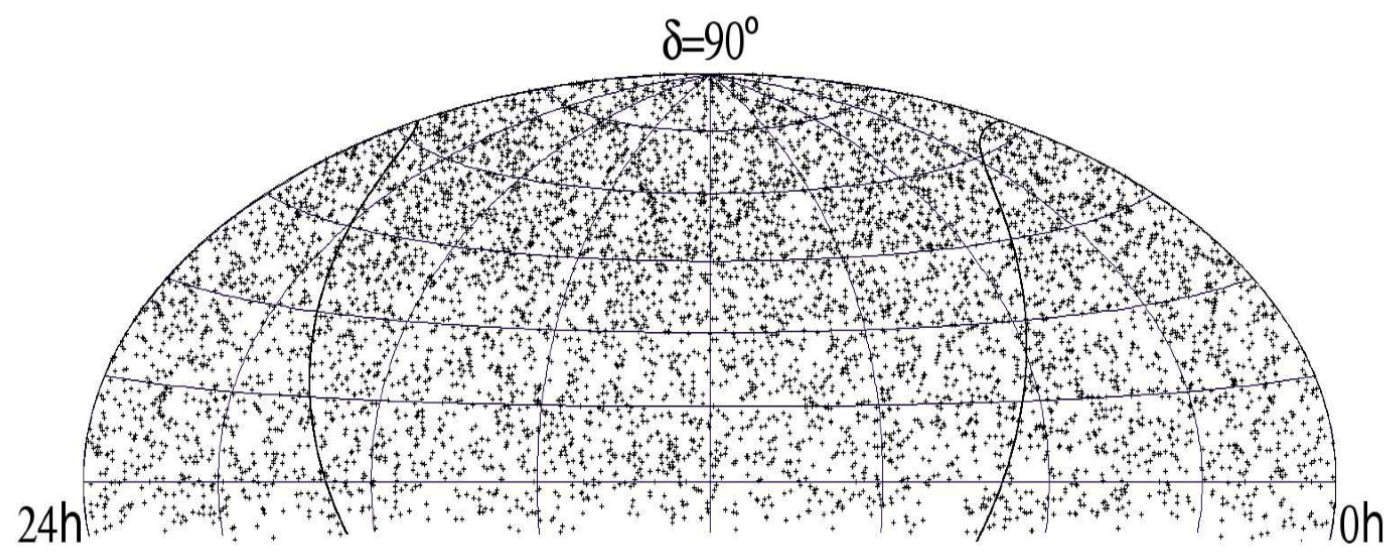

Figure 4: Equatorial sky map of 6595 events recored by AMANDA-II from 2000-2007 [6].

The AMANDA-II detector ran successfully until May 2009 when it was shut down. In total almost 10000 atmospheric neutrinos were recorded but no neutrinos were positively identified to be extraterrestrial. The equatorial sky map of 6595 events recorded during 2000-2006 with AMANDA-II is shown in Figure 4. The detector completely dominated the field of neutrino astronomy and gave the most sensitive limits for the flux of extraterrestrial neutrinos.

\section{IceCube}

We realised very soon that AMANDA would not be large enough to get high statistics of extraterrestrial neutrinos. A new much larger detector, IceCube, was designed aiming for a total 
mass of the order of one gigaton (one cubic kilometer of ice).

The IceCube Neutrino telescope design was based on the experience from AMANDA. It was completed in December 2010 and is the first neutrino telescope to reach the expected necessary gigaton scale. It consists of 86 strings with 60 Digital Optical Modules (DOM) deployed between 1450 and $2450 \mathrm{~m}$ depth. The Stockholm University and Uppsala University groups built and tested 1050 of the 5160 DOMs. A cosmic ray air-shower array, IceTop, is situated at the snow surface with high efficiency for PeV - EeV energies. An IceTop station is placed close to each string hole and consists of two tanks with frozen water with two DOMs each. The combination of the neutrino telescope and the air-shower telescope is a unique feature of the South Pole installation. It gives the possibility for absolute direction calibration of the neutrino telescope as well as for studies of the chemical components of the air-showers. The deployment of the IceCube modules is done as in AMANDA but with a much more efficient hot water drill. A $2450 \mathrm{~m}$ deep hole is completed within less than $35 \mathrm{~h}$ (compare with $90 \mathrm{~h}$ for a $1000 \mathrm{~m}$ hole for AMANDA-A) and the deployment of the string takes less than $10 \mathrm{~h}$. The hole is completely frozen after about two weeks. The drilling and deployment is only possible during the austral summer, November - February.

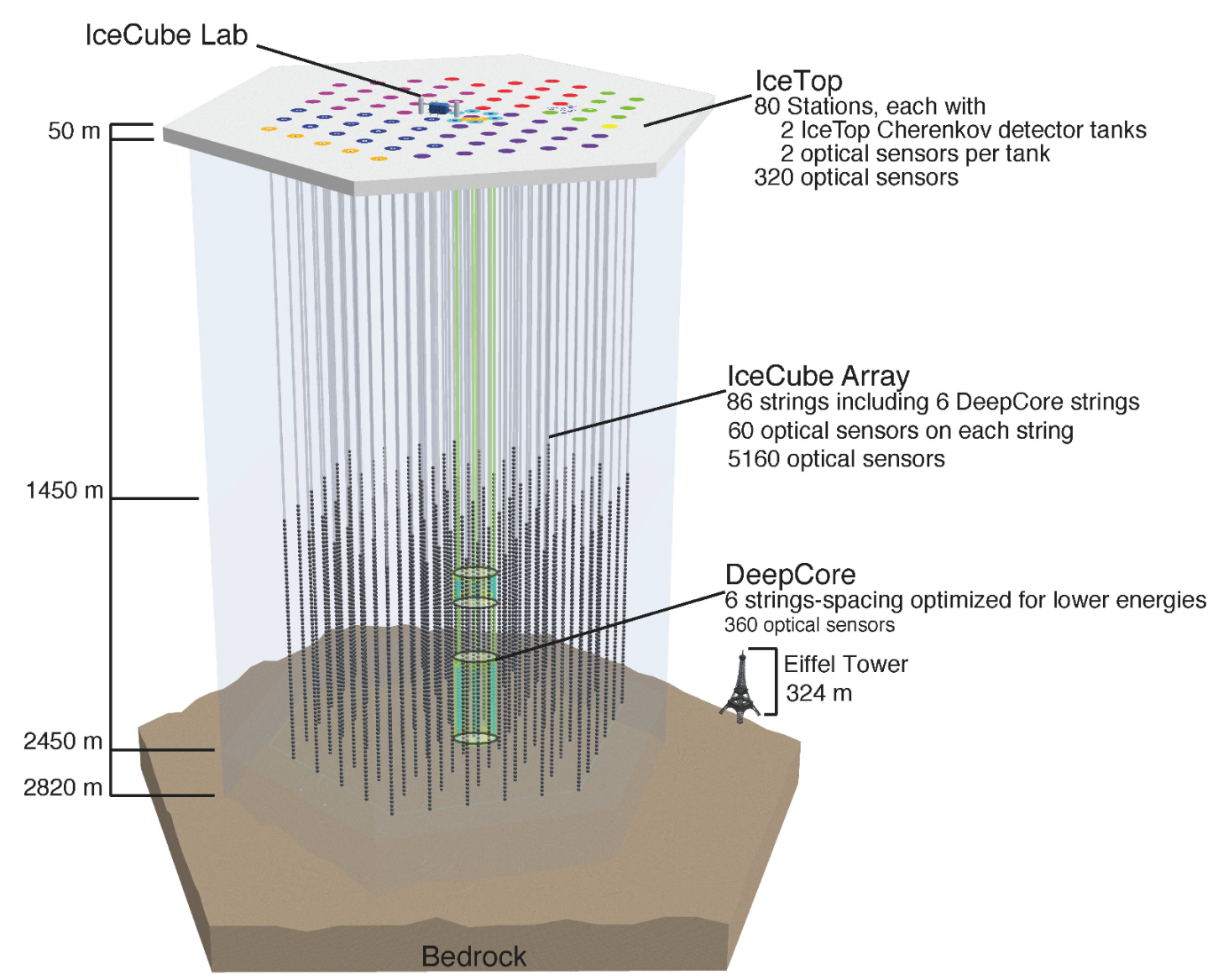

Figure 5: The IceCube neutrino telescope with the air-shower IceTop on surface and the new DeepCore low energy sub-detector.

Figure 5 shows the IceCube neutrino telescope with the air-shower array IceTop on surface. 
IceCube was originally designed with 80 strings with a spacing of $125 \mathrm{~m}$. The Swedish groups proposed to add six strings in the centre of the array giving a central part of IceCube with $72 \mathrm{~m}$ spacing between 13 strings. This sub detector is called DeepCore and it will improve the threshold for low energy neutrinos. By using the surrounding IceCube strings as active veto for incoming atmospheric muons it will open up the Southern Sky for low energy neutrinos. The K\&A Wallenberg Foundation approved very quickly an application for DeepCore of $2.8 \mathrm{M} \$$. The Belgian and German groups secured additional funding for DeepCore which gave the possibility to increase the number of optical modules and buying photomultipliers with higher quantum efficiency. The DeepCore strings are equipped with 50 High Quantum efficiency (HQE) DOMs with seven metre spacing (compared with $17 \mathrm{~m}$ for standard IceCube strings) between depths of $2107 \mathrm{~m}$ and 2450 $\mathrm{m}$ where the ice is most transparent and $10 \mathrm{HQE}$ DOMs with $10 \mathrm{~m}$ spacing between $1750 \mathrm{~m}$ and $1840 \mathrm{~m}$. See Figure 5.

IceCube has been taking data with the partially completed telescope every year since 2005 (with 1, 9, 22, 40, 59 and 79 strings configurations called IC1, IC9, IC22, IC40, IC59 and IC79). In total about 20000 up-going muon neutrinos have been analysed in data taken up to and including 2008 (IC40). The median angular resolution is expected to be less than $0.5^{\circ}$ for the final detector.

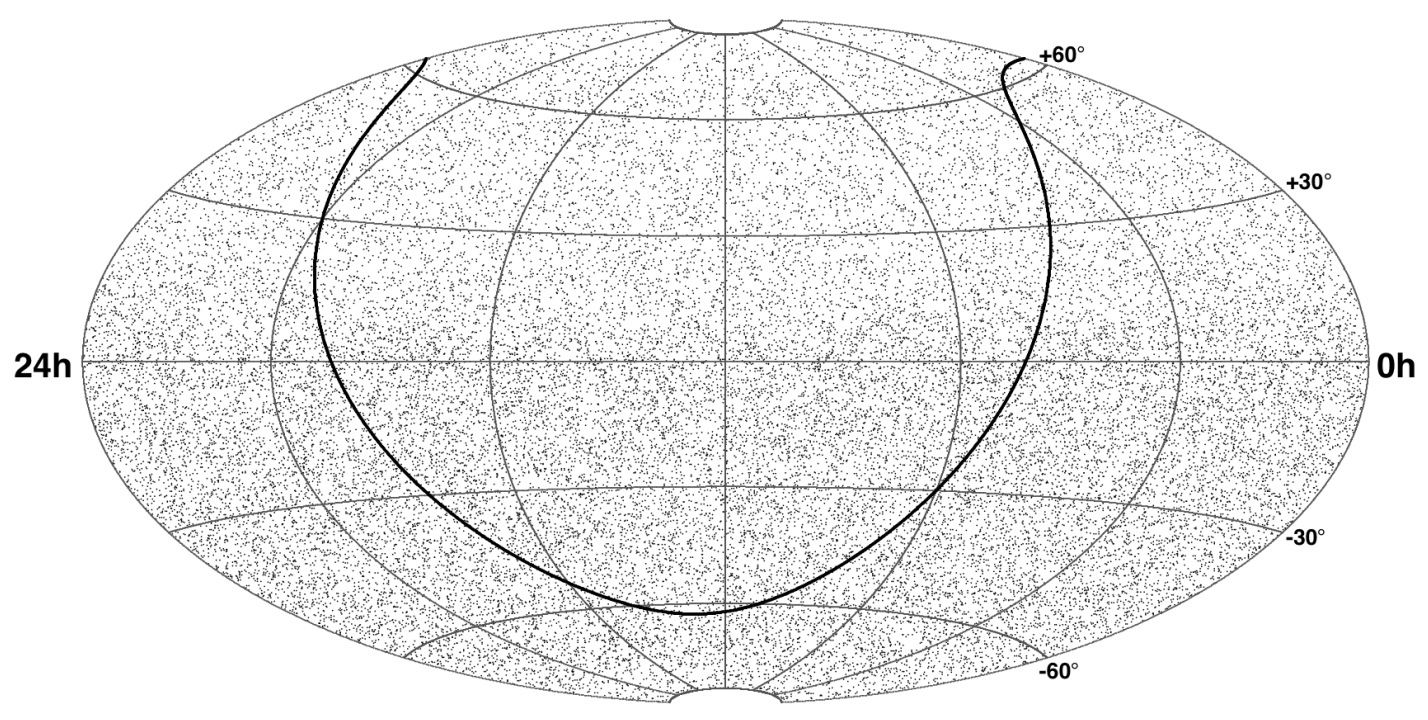

Figure 6: Equatorial skymap showing the 36,900 events in the final IC40 sample. The galactic plane is shown as the solid black curve. The northern sky (positive declinations) is dominated by up-going atmospheric neutrino-induced muons, and the southern sky (negative declinations) is dominated by muons produced in cosmic ray showers in the atmosphere above the South Pole.

Figure 6 shows the sky map in equatorial coordinates for the point source analysis of IC40 [7]. The northern hemisphere with 14000 events is mainly up-going atmospheric muon neutrinos. The southern hemisphere with 22700 events is mainly muons from cosmic ray interactions in the atmosphere above the detector. We have here reduced the background of down going atmospheric muons by applying an energy cut assuming that the cosmic neutrinos have a harder energy spectrum 
than the atmospheric muons. The down going flux of atmospheric muons is reduced by a factor $10^{-5}$ in Figure 6. No significant point source could be identified in this data..

One year of datataking with the IC40 detector (50\% of the final IceCube detector size) is by itself more than two times more efficient than seven years data taking with the AMANDA detector.

The IceCube detector is now a complete one gigaton detector which holds the promise of finally opening the field of Neutrino Astronomy. The coming years will be very interesting. Sweden is heavily involved in this field and this is to no small degree thanks to Héctor Rubinstein. The size of the IceCube neutrino telescope is more than 600 times larger than the detector we discussed to deploy in Torne Träsk.

\section{Summary}

Héctor Rubinstein played an important role for the start of the Swedish involvement in the new field of Neutrino Astronomy and it is very sad that he is not able to follow the exciting time we now are entering. We miss him very much.

\section{References}

[1] A. Adams et.al. The Grande Proposal (1990), UCL

[2] H. Sobel Neutrino Telescopes Nuclear Physics B Proc. Suppl. 28A (1992)

[3] D.M. Lowder et al Observation of muons using the polar ice cap as a Cerenkov detector. Nature $\mathbf{3 5 3}$ (1991)

[4] P. Askebjer.,et.al.Optical Properties of the South Pole ice at Depth between 0.8 and 1 kilometer Science 267 (1995) 1147

[5] E. Andres et al. Observation of high-energy neutrinos using Cherenkov detectors embedded deep in Antarctic ice, Nature 440 (2001) 441

[6] R. Abbasi,et al. Search for Point Sources of High Energy Neutrinos with Final Data from AMANDA-II, Phys Rev D79 (2009) 062001

[7] R. Abbasi,et al. Time-Integrated Searches for Point-like Sources of Neutrinos with the 40-String IceCube Detector, 1012.2137 [astro-ph. HE] Submitted to Phys Rev D. 